Presented at the American College of Osteopathic Internists annual meeting, September 28, 1987. Supported by an educational grant from Merck Sharp and Dohme. See related editorial on page 843.

\title{
Detection, evaluation, and treatment of hypercholesterolemia in adults: Commentary on the National Cholesterol Education Program guidelines
}

\author{
W.H. VOSS, DO, FACOI
}

After the value of intervention to lower serum cholesterol levels was conclusively demonstrated, principally by the Lipid Research Clinics Coronary Primary Prevention Trial, the National Institutes of Health convened a conference to produce a consensus on ways to achieve this. As a result, the National Cholesterol Education Program has developed guidelines for detecting, classifying, and treating hypercholesterolemia in adults; these are summarized in the article. The program focuses on a patient-based approach. It classifies total cholesterol values as desirable, borderline, or high, and identifies who should have lipoprotein analysis and who should undergo cholesterol-lowering treatment. The dietary approach described is the main treatment for lowering cholesterol values, with drug treatment reserved for those in whom a trial of diet therapy fails to achieve the low density lipoprotein cholesterol goal.

The role of elevated serum cholesterol levels in the etiology of atherosclerosis was strongly suspected for many decades on the basis of epidemiologic, pathologic, and animal research data. Nonetheless, this theory was exceedingly difficult to prove. It was more difficult still to show that lowering cholesterol levels reduced mortality from coronary heart disease (CHD). As a result, the medical profession has not shown a united front to the public on the cholesterol question, and many physicians themselves have remained unconvinced.

In recent years, however, a series of large prospective trials have shown beyond reasonable doubt that an elevated serum cholesterol level leads to CHD. Some of these trials also have been able to demonstrate that intervention to lower a person's serum cholesterol level slows or reverses the pathologic processes involved in atherogenesis and reduces the person's risk of $\mathrm{CHD}$ and the other lifethreatening sequelae of atherosclerosis.

The most recent of these studies was the Lipid Research Clinics (LRC) Coronary Primary Prevention Trial (CPPT). ${ }^{1-3}$ This investigation, which involved more than 3,800 hypercholesterolemic men, was the first to show conclusively the value of intervention in lowering cholesterol levels. The clearcut results were the primary catalysts for the convening of the Consensus Development Conference on Lowering Blood Cholesterol to Prevent Heart Disease. This conference was held in late 1984 at the National Institutes of Health $(\mathrm{NIH})$ and yielded a consensus report ${ }^{4}$ published in 1985 .

\section{National Cholesterol Education Program}

The NIH consensus report might fairly be called the first unified statement of the medical community on the cholesterol issue. Out of it have come guidelines ${ }^{5}$ for detecting, classifying, and treating hypercholesterolemia in adults that will affect tens of millions of Americans and have a major impact on the US health-care system. This paper includes a brief synopsis of these guidelines.

Among the consensus panel's recommendations was that NIH develop a plan for a National Cholesterol Education Program (NCEP), which would parallel similar programs in hypertension and smoking. This program, according to the panel's mandate, "would educate physicians, other health professionals, and the public to the significance of elevated blood cholesterol levels and the importance of treating them. [The program] would enlist par- 
ticipation by and contributions from all interested organizations at national, state, and local levels."4

The education program was duly established under the auspices of the National Heart, Lung, and Blood Institute (NHLBI). Translated into specific action, its mandate was first, to bring to treatment those now at significant risk of CHD and, second, to promote the lowering of the range of serumcholesterol levels across the entire US population. This seemed reasonable and fairly straightforward at first glance. However, to carry it out has required that an expert panel of the NCEP grapple with some of the most difficult and controversial questions in medicine today.

For example, no one disputes that CHD is still the leading cause of mortality and morbidity in the United States, that high levels of serum cholesterol are clearly linked to CHD, that elevation of serum cholesterol levels is virtually epidemic in the US population, and that intervention to lower cholesterol levels is justified. ${ }^{6}$ However, many experts in the field hold that hypercholesterolemia is not a proper province for public-health approaches, such as NHLBI's general recommendation that everyone lower the intake of fats to $30 \%$ of calories consumed. They would prefer to manage hypercholesterolemia with a patient-based approach, using individually planned therapy, and to make no sweeping recommendations for dietary change. These issues will necessarily continue under discussion.

\section{Adult-treatment recommendations}

The guidelines from NCEP's expert panel take a patient-based approach. However, they are complementary to NCEP's public health strategy, which is designed to lower cholesterol levels in the general US population.

This strategy is considered to be vital. At present, if the guidelines to detect and treat those presently at risk from hypercholesterolemia are followed, tens of millions of new patients will be directed into a health-care system that already must husband its resources closely. If the public health effort is successful, however, fewer persons will have to be treated, and the country more likely will be able to afford the patient-based part of the program.

The expert panel's report represents a continuation of the original consensus process, in that it consists of a working resolution of a number of the debates surrounding the issues of whom to treat for elevated blood cholesterol levels, how to treat them, and when. Although these issues may not have been fully resolved to the satisfaction of everyone working in the field, the medical profession cannot afford to stand still in this pressing area, and the guidelines provide it a way of moving forward usefully.

\section{Summary of the guidelines}

The principal areas dealt with in the panel report are detection of cases, criteria for treatment decisions, dietary therapy, and drug therapy. There are three main sections and four appendixes in the document, as follows:

(1) Detection, classification, and evaluation. Among the topics discussed in this section are the rationale for intervention, persons who should be tested, methods of sample collection, patient classification by total cholesterol and low density lipoprotein (LDL) cholesterol levels, and clinical evaluation.

(2) Dietary treatment. This section covers goals and cutpoints for dietary therapy, recommended step-one and step-two diets (including the roles of saturated and unsaturated fats, dietary cholesterol, fiber, alcohol, protein, carbohydrates, and total calories), setting up a diet program for patients, monitoring and follow up, special diets (in pregnancy, old age, and severe lipid disorders), and the dietary approach for the borderline high LDL group.

(3) Drug treatment. The final section addresses the cutpoints for initiating drug therapy, the target levels of LDL cholesterol to be achieved by treatment, first-choice drugs, the newer pharmacotherapies (such as hydroxymethylglutaryl coenzyme A [HMG-CoA]-reductase inhibitors), other drugs, combination drug therapies, monitoring and follow up; and problems of patient compliance.

(4) Appendixes. These include the following: total and LDL cholesterol distributions in the United States; patient populations with special disorders (including familial hypercholesterolemia, severe primary hypercholesterolemia, familial dysbetalipoproteinemia, hypertriglyceridemia, reduced high density lipoprotein [HDL] cholesterol level, diabetic dyslipidemia, and lipid disorders in pregnancy); intensive dietary and behavior modification; and suggestions for improving patient compliance with drug therapy.

\section{Detection}

The first tasks are to detect patients at risk of CHD from elevated blood cholesterol levels, to classify them according to severity of risk, and then to route them appropriately to simple dietary counseling, intensive dietary therapy, or dietary therapy combined with drug therapy. Thus, before we reach the hard questions of what are appropriate dietary and pharmacologic measures for hypercholesterolemia, we must decide what really constitutes elevated blood cholesterol levels in the context of CHD risk, and then establish cutpoints for the various cate- 


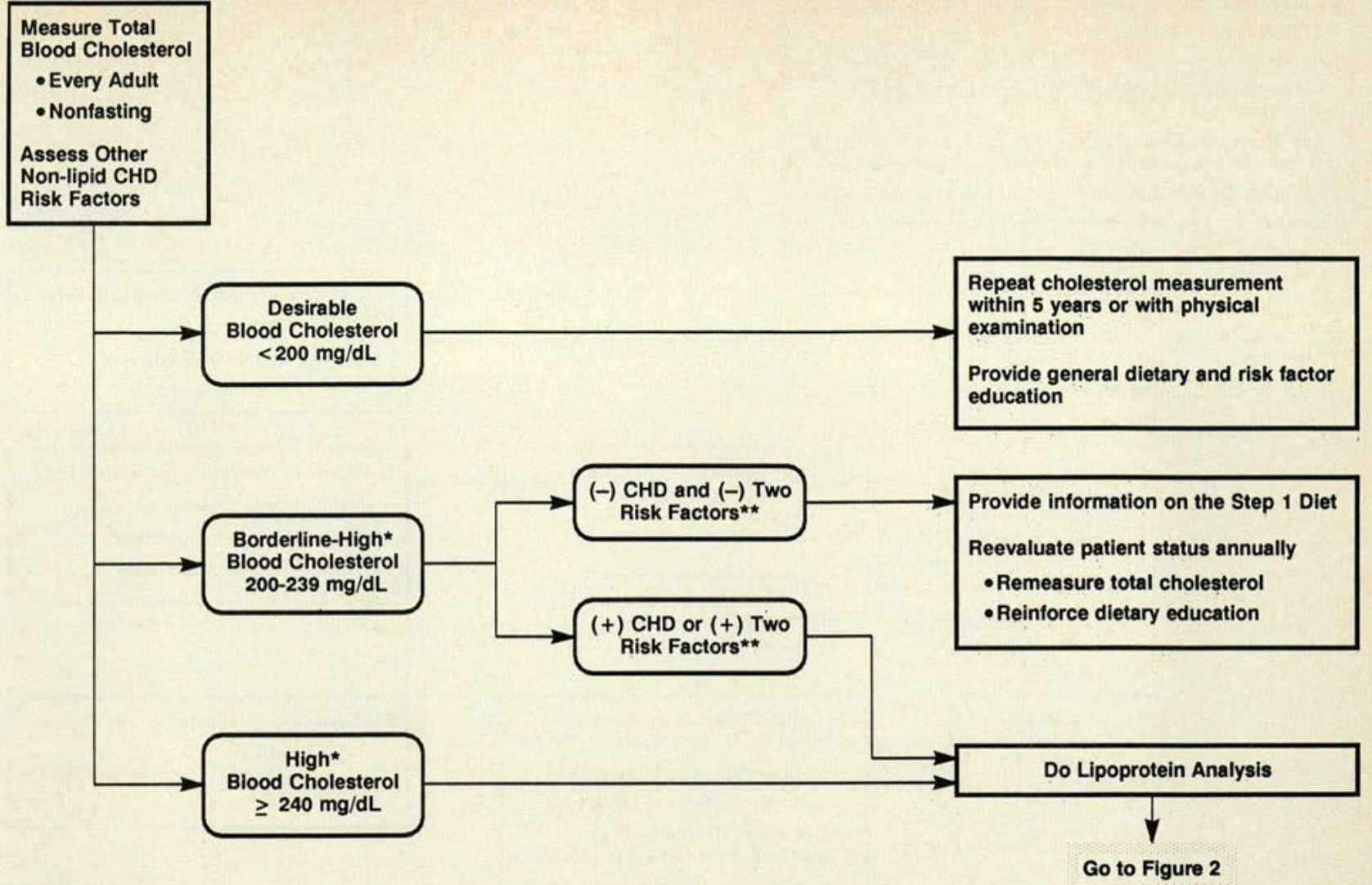

Fig 1. Initial classification based on total cholesterol level. Asterisk indicates must be confirmed by obtaining repeated measurements and then using the average value; double asterisks indicate one of which can be male sex. Reprinted from Archives of Internal Medicine (1988;36-69).

gories of severity. Because the cholesterol risk curve is continuous, cutpoints are somewhat arbitrary, but nonetheless necessary.

While LDL cholesterol is the fraction of total cholesterol that is directly related to CHD risk, total cholesterol is easier and less expensive to assay, and does not require the patient to fast before the blood sample is drawn. Because LDL cholesterol constitutes $60 \%$ to $70 \%$ of a person's total cholesterol, the two are well correlated. This, in accordance with the guidelines, total cholesterol may be used as a surrogate parameter for the LDL cholesterol when mass testing is contemplated and great accuracy is not required. Patients whose total cholesterol levels put them in the high-risk groups are asked to return for a fasting lipoprotein analysis to determine their LDL cholesterol levels and, thus, their appropriate therapeutic categories. Retesting for LDL cholesterol is especially important when drug therapy is contemplated.

The cutpoint between normal or desirable total cholesterol levels and borderline high levels has been set at $200 \mathrm{mg} / \mathrm{dL}$ for both men and women. Between borderline high and high values, the cutpoint is $240 \mathrm{mg} / \mathrm{dL}$. Above the latter value, the risk of CHD rises steeply. On the other hand, the benefit of cholesterol reductions in this group is correspondingly great; for every $1 \%$ reduction in total cholesterol, there is a $2 \%$ reduction in CHD risk. ${ }^{3}$ The high-risk LDL cholesterol cutpoint is $160 \mathrm{mg} /$ dL. According to these guidelines, $25 \%$ of adults in the United States can be categorized as being at high risk. (This percentage gives an idea of the magnitude of the effort called for.)

The guidelines call for all adults over 20 years of age to be tested for total cholesterol at least every 5 years. This can be done by means of periodic screening programs, if these use accepted cholesterol assay methods and include proper follow up of patients with high levels. Usually, however, the total cholesterol test will be included in regular physical examinations and, when appropriate, in other medical examinations. The suggested testing methodology is described in the guidelines.

Patients whose total serum cholesterol value is below the $200-\mathrm{mg} / \mathrm{dL}$ level should be given general information on diet and risk factors when they are told their test results. Those with higher values should be retested within 8 weeks. If the two values are within $30 \mathrm{mg} / \mathrm{dL}$ of one another, they should 


\begin{tabular}{|l|}
\hline Do Lipoprotein Analysis \\
-12-hour fast \\
- Measure total cholesterol, HDL-cholesterol, and \\
triglycerides \\
- Estimate LDL-cholesterol = \\
total cholesterol - HDL-cholesterol - triglycerides/5 \\
-Average of 2 to 3 measurements, 1 to 8 weeks apart
\end{tabular}

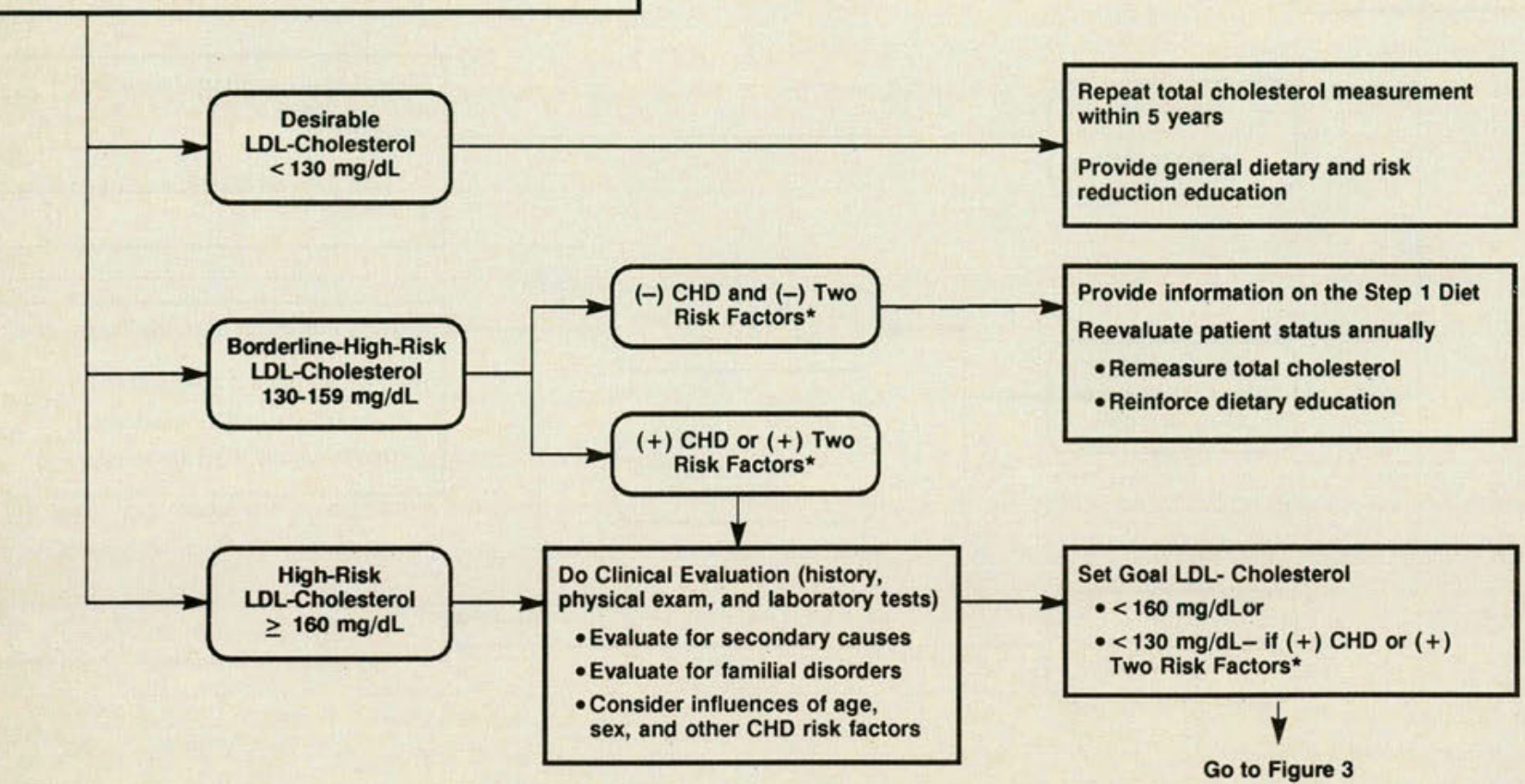

Fig 2. Classification based on LDL cholesterol value. Asterisk indicates one of which can be male sex. Reprinted from Archives of Internal Medicine (1988;36-69).

be averaged. If they differ by more than this, they should be averaged along with the result of a third test, which should be performed within another 8 weeks. This retesting is very important, because total cholesterol levels may fluctuate widely from day to day.

Patients in the borderline high group may follow one of two paths (Fig 1), with the choice depending on additional risk factors. They may be advised to go on the step-one diet, which will be described later, and asked to come in for annual retesting for total cholesterol. Or they may join those in the high-risk group (total cholesterol $>240$ $\mathrm{mg} / \mathrm{dL}$ ) and undergo repeated fasting lipoprotein analyses to determine the LDL cholesterol level.

Patients whose LDL cholesterol is in the normal range $(<130 \mathrm{mg} / \mathrm{dL})$ will receive the same general counseling given to those with total cholesterol $<200 \mathrm{mg} / \mathrm{dL}$ (Fig 2). Patients with borderline results ( 130 to $159 \mathrm{mg} / \mathrm{dL}$ ) will either be advised to follow the step-one diet (if they have less than two risk factors) or will undergo a full clinical evaluation along with those patients falling into the highrisk group (LDL cholesterol $>160 \mathrm{mg} / \mathrm{dL}$ ).

The panel considered the major risk factors to be existing CHD, male sex, family history of premature CHD (before age 55), smoking, hypertension, low level of HDL cholesterol, diabetes, history of stroke or other occlusive vascular disease, and severe obesity. These factors determine the treatment pathway followed by patients with borderline high cholesterol levels, and they must be carefully evaluated in those persons selected for clinical appraisal.

\section{Clinical evaluation}

The clinical evaluation is intended to determine whether the hypercholesterolemia is secondary to drugs or other diseases, whether there are genetic contributions if the disorder is primary, and what the actual clinical consequences of risk factors (such as older age and smoking) are. It should include a history, physical examination, and the usual laboratory tests. In light of the clinical evaluation, a treatment target level of LDL cholesterol should be set, and the patient should be enrolled in an appropriate program of therapy.

Secondary elevations in LDL cholesterol may be caused by a number of drugs, especially progestins and anabolic steroids, or by certain diseases. These 
include hypothyroidism, diabetes, obstructive liver disease, and nephrotic syndrome. If the clinical examination reveals one of the foregoing, the guidelines recommend treating the disease or withdrawing the drug, then retesting for LDL cholesterol.

Among patients with primary hypercholesterolemia, a significant number will have one of a number of heritable disorders described in Appendix II of the guidelines. These include familial hypercholesterolemia $(\mathrm{FH})$ and familial combined hyperlipidemia (FCHL). Patients with some of the severe, genetically determined hyperlipidemias are best referred to specialists.

The evaluation must consider the roles of other lipid risk factors. The guidelines are cautious on the significance and treatment of reduced serum HDL cholesterol. Although HDL cholesterol is a strong inverse risk factor for CHD, there is no evidence from clinical trials that intervention to raise HDL cholesterol levels will actually reduce CHD mortality-somewhat the same state of affairs that existed until recently with total cholesterol and LDL cholesterol.

Among the principal causes of low HDL cholesterol levels, however, are smoking, obesity, lack of exercise, the use of anabolic steroids, and hypertriglyceridemia. In addition, heavy coffee use may exacerbate the HDL cholesterol reduction caused by smoking. While awaiting more definite evidence on the value of intervention to raise HDL cholesterol values, the panel recommends that patients reduce or eliminate all these factors, on the strength of the HDL cholesterol link to CHD and because in themselves many are CHD risk factors. The use of either the LDL cholesterol:HDL cholesterol ratio or the total cholesterol:HDL cholesterol ratio in the evaluation is not recommended, even though these ratio are often advocated as better indexes of CHD risk than an LDL, HDL, or total cholesterol measurement taken alone. ${ }^{7}$ The panel thought that physicians should weigh HDL cholesterol and LDL cholesterol separately in making their treatment decisions, because the two are independent risk factors arising from different causes. Thus, a combination number should not be used, any more than a blood pressure:smoking data ratio would be used.

The Framingham study found that hypertriglyceridemia is an independent CHD risk factor. ${ }^{8}$ However, most other studies have not been able to demonstrate this after making necessary statistical adjustments for associated factors such as obesity, hypertension, and elevated total cholesterol level. Furthermore, many diseases that elevate triglyceride levels also increase the risk for $\mathrm{CHD}$, and it is probable that it is these conditions that impose the risk, not the triglycerides per se. In certain circum- stances, an elevated triglyceride level may serve as a marker for underlying lipoprotein derangements having a more definite connection with CHD.

Whatever the role of triglycerides in CHD, the issue is unresolved and controversial. The guidelines defer for suggestions on management of this disorder to the report of the NIH Consensus Development Conference on treatment of hypertriglyceridemia, ${ }^{9}$ which is summarized in Appendix II of the guidelines. Intervention directed specifically at triglycerides is not recommended, except in the rare case when there is a risk of pancreatitis. The physician should keep in mind, though, that many persons with elevated triglyceride values also have a low level of HDL cholesterol, elevated LDL cholesterol value, or both.

The influences of the non-lipid risk factors mentioned previously must also be weighed carefully during the clinical evaluation. This is especially true of risk factors that can be modified, such as smoking and hypertension. (Advice on intervention in hypertension and smoking is found in The 1984 Report of the Joint National Committee on Detection, Evaluation, and Treatment of High Blood Pressure, to be updated in 1988 and Clinical Opportunities for Smoking Intervention: A Guide for the Busy Physician, 1986, both published by the National Heart, Lung, and Blood Institute.) In a given patient, these may be as important as targets for intervention as is the elevated cholesterol level itself. In addition, the therapy used to modify these factors must be integrated with the treatment for the hypercholesterolemia. A number of drugs used in hypertension affect the lipid profile, for example.

The guidelines take the position that even the unmodifiable risk factors such as age and sex must play an important part in treatment decisions, because they greatly increase a patient's absolute risk of CHD, as distinct from the relative or percentage risk. In the Multiple Risk Factor Intervention Trial (MRFIT), ${ }^{10}$ for example, a move from the first to the fifth quintile of total cholesterol incurred an additional 4.8 deaths per 1,000 in men without other risk factors (Table 1). The same move among men with two other risk factors, in contrast, incurred an additional 15.1 deaths. Thus, for the latter group of patients, a total cholesterol reduction from the fifth to the first quintile would be three times more worthwhile than for the former group, in terms of lives saved by the cholesterol-lowering intervention alone.

Clearly, therefore, much more aggressive therapy is justified in the presence of added risk factors. Such factors are especially important when considering the benefit-risk ratios of drug therapy. For example, in middle-aged men, whose CHD rate 
TABLE 1. CORONARY HEART DISEASE DEATHS PER 1000 IN MEN 35 TO 57 YEARS OF AGE WITH AN AVERAGE FOLLOWUP OF SIX YEARS ACCORDING TO SERUM CHOLESTEROL QUINTILE AND PRESENCE OR ABSENCE OF OTHER RISK FACTORS. THE DIFFERENCE IN ABSOLUTE RISK IN THE HIGHEST VS THE LOWEST QUINTILE OF SERUM CHOLESTEROL IS GREATER IN PATIENTS WHO ARE AT HIGH RISK FOR OTHER REASONS.*

\begin{tabular}{ccc}
$\begin{array}{c}\text { Serum Cholesterol } \\
\text { Quintile, mg/dL }\end{array}$ & $\begin{array}{c}\text { Normotensive } \\
\text { nonsmoker }\end{array}$ & $\begin{array}{c}\text { Hypertensive } \\
\text { smoker }\end{array}$ \\
\hline$<82$ & 1.6 & 6.3 \\
182 to 202 & 2.5 & 10.0 \\
203 to 220 & 2.7 & 15.5 \\
221 to 244 & 3.8 & 16.6 \\
$\geqslant 245$ & 6.4 & 21.4
\end{tabular}

*Reprinted from Archives of Internal Medicine (1988; 36-69).

is three to four times greater than that of their female counterparts, ${ }^{11}$ a drug that is contraindicated in women with the same LDL cholesterol levels may be strongly indicated.

The reports acknowledges the factor of age to be difficult to assess. The LDL cholesterol cutpoints used in the guidelines- $160 \mathrm{mg} / \mathrm{dL}$ for high risk and $190 \mathrm{mg} / \mathrm{dL}$ for very high risk-correspond respectively to the 75 th and 90 th percentiles for patients 35 to 60 years old. For younger patients, however, these values are too high. Using them may cause many at-risk patients to be given inadequate treatment, when in fact these persons should be especially sought out to arrest atherogenesis at the earliest possible stage. Dietary modification, however, should be the focus, rather than drug treatment, whose long-term safety is not known. At the other end of the spectrum, the elderly patient may be overtreated.

There is less connection in elderly patients between LDL cholesterol and CHD. There is also little direct evidence for the benefit of intervention in relative terms, although the absolute magnitude of benefits is significant because of the coexisting risk of CHD. ${ }^{12}$ To remedy this, the guidelines include the cholesterol percentiles for various age groups in Appendix I. Although the recommendations are intended to apply generally to all adults 20 years of age and older, the information in the appendix should be used during the clinical evaluation to modify the approach as necessary for patients at either end of the age spectrum.

\section{Dietary therapy}

Diet is the first option among the treatment regimens proposed in the guidelines and remains central even if it finally becomes necessary to resort to drug therapy. In summary, the dietary recommendations are as follows:

General approach. Patients selected for dietary therapy as described and having no additional risk factors should set $160 \mathrm{mg} / \mathrm{dL}$ as the highest level of LDL cholesterol acceptable. If there is definite CHD or two other risk factors, the target should be no higher then $130 \mathrm{mg} / \mathrm{dL}$. It should be emphasized that these are the minimal goals, and lower levels of LDL cholesterol should be reached if possible to achieve the greatest possible reductions in risk. In most patients, total cholesterol measurements can be used as surrogates for LDL cholesterol for purposes of monitoring responses to diet, using total cholesterol levels of 240 and $200 \mathrm{mg} / \mathrm{dL}$ for LDL cholesterol levels of 160 and $130 \mathrm{mg} / \mathrm{dL}$, respectively. Of course the surrogate parameter should not be used as the end-point for therapy, nor in patients with hypertriglyceridemia or abnormal HDL cholesterol levels, in whom the usual correlation between total cholesterol and LDLcholesterol may not exist.

High intakes of saturated fats, cholesterol, and total calories are the three dietary factors most likely to raise serum cholesterol levels. Therefore, they are the three targets at which the guidelines' dietary modifications are aimed most directly. The basic approach is similar to that advocated by the American Heart Association and others, but it is used in a context of medical care, intensive counseling, and laboratory monitoring of results that justifies the use of the term dietary therapy. $\mathrm{Pa}$ tients should be convinced that the dietary changes are permanent alterations in life-style rather than short-term abstentions.

Step-one and step-two diets. Two diets are outlined in the report (Table 2). The step-one diet can be directed by the physician and office staff. It seeks to eliminate or cut back the more obvious sources of saturated fats and cholesterol, and may not require major shifts in the patient's dietary habits. It stipulates a saturated fatty-acid intake of $10 \%$ or less of total calories, total fat reduction to less than $30 \%$ of calories, and less than $300 \mathrm{mg}$ of dietary cholesterol per day.

If the patient has not achieved the target LDL cholesterol level after three months on the stepone regimen, the more stringent measures of the step-two diet should be started; fat intake still less than $30 \%$ of total calories, but saturated fatty acid intake now is cut to $7 \%$ or less, and cholesterol intake is reduced to less than $200 \mathrm{mg} /$ day. This regimen is probably best supervised by a registered dietitian. Supervision involves reviewing the entire diet for relatively minor contributions of saturated fat and cholesterol, then minimizing these contributions while still providing a nutritious, balanced diet.

Total fat may be reduced in the step-two diet to as little as $20 \%$ of total calories for weight loss or for further decreases in saturated fat intake. How- 
TABLE 2. DIETARY THERAPY OF HIGH BLOOD CHOLESTEROL LEVEL ${ }^{*}$

\begin{tabular}{lll}
\hline & \multicolumn{2}{|c|}{ Recommended intake } \\
Nutrient & Step-One diet & Step-Two diet \\
\hline Total fat & Less than $30 \%$ of total calories & Less than $30 \%$ of total calories \\
$\quad$ Saturated fatty acids & Less than $10 \%$ of total calories & Less than $7 \%$ of total calories \\
Polyunsaturated fatty acids & Up to $10 \%$ of total calories & Up to $10 \%$ of total calories \\
$\quad$ Monounsaturated fatty acids & $10 \%$ to $15 \%$ of total calories & $10 \%$ to $15 \%$ of total calories \\
Carbohydrates & $50 \%$ to $60 \%$ of total calories & $50 \%$ to $60 \%$ of total calories \\
Protein & $10 \%$ to $20 \%$ of total calories & $10 \%$ to $20 \%$ of total calories \\
Cholesterol & Less than 300 mg/d & Less than 200 mg/d \\
Total calories & To achieve and maintain desirable weight & To achieve and maintain desirable weight \\
"Reprinted from Archives of Internal Medicine (1988; $36-69)$ & \\
\hline
\end{tabular}

ever, total fat reductions below $30 \%$ are not necessary for adequate lowering of LDL cholesterol levels, provided that most or all of the fat consumed is unsaturated. Overenthusiastic restriction of total fat may reduce the palatability of the diet to the point of hindering patient compliance.

Nutrients. The report discusses sources and dietary roles of cholesterol and of saturated, polyunsaturated, and monounsaturated fatty acids. Cholesterol is found in almost equal concentrations in muscle tissue and fat, based on wet weight. As is well known, it is richly supplied by egg yolks, organ meats, and some shellfish. Saturated fatty acids are abundant in animal-derived fats, coconut oil, palm oil, and palm-kernel oil. Neither cholesterol nor saturated fat is required in a healthy diet.

Polyunsaturated fatty acids are found in vegetable fats, such as safflower, sunflower seed, soybean, and corn oils, and in fish oils. They have a role in lowering serum cholesterol, but because the effects of long-term consumption of these oils are unknown, an upper dietary limit of $10 \%$ of calories is generally recommended. Some monounsaturated fatty acids appear to possess the same cholesterollowering ability as the polyunsaturates. Occurring in olive, peanut, rapeseed, and certain other oils, they are considered safer than polyunsaturates; they have been consumed for centuries or millennia by certain populations known for low incidences of CHD. Monounsaturates should provide $10 \%$ to $15 \%$ of calories on both the step-one and step-two diets.

Between $50 \%$ and $60 \%$ of calories in both diets should come from carbohydrates, with more than half of the digestible carbohydrates in the form of complex carbohydrates. Adequate fiber is important to normal bowel function, but the insoluble fibers, such as are found in wheat bran, appear to have almost no effect on blood cholesterol. On the other hand, soluble fibers, such as are found in oat bran and psyllium, have cholesterol-lowering ability. Expected responses. The response to dietary ther- apy varies considerably because of inherent metabolic factors, environmental constraints, and prior eating habits. As a rule, larger absolute gains can be realized in patients who start therapy with high LDL cholesterol levels than in those with lower levels, as might be expected. The guidelines cite studies in metabolic wards showing that average reductions in total cholesterol of 30 to $40 \mathrm{mg} / \mathrm{dL}$ can be achieved by adherence to the step-one diet in place of the "typical" American fare. An additional reduction of about $15 \mathrm{mg} / \mathrm{dL}$ results from moving to the step-two regimen. The physician should not hasten to drug therapy if diet does not yield quick results. A minimum of six months should be allowed before deciding that a patient requires drugs as well as diet, except in persons who are at very high risk of CHD from severe dyslipidemia.

Practical approach. This section of the report details the various roles of the physician, the office staff, and the registered dietitian. Changes in dietary patterns are suggested, such as the use of egg whites for egg yolks and sherbet for ice cream. Recommendations are made for each basic food group, for cooking methods, for eating away from home, and for successful behavioral modification leading to permanent changes in eating habits. A plan is provided for monitoring progress on each diet and following up as necessary with additional therapy when response is inadequate (Fig 3 ).

Special groups. Two sections are devoted to dietary therapy for special groups. The first is devoted to diets in severe dyslipidemias, old age, and pregnancy. The second discusses the dietary therapy required by the borderline high LDL group.

\section{Drug therapy}

Pharmacotherapy of hypercholesterolemia is discussed in detail elsewhere in this issue. ${ }^{13}$ Here I will touch briefly on a few areas of special concern in the report.

First, the guidelines predicate the initiation of drug therapy on the patient's having received at 


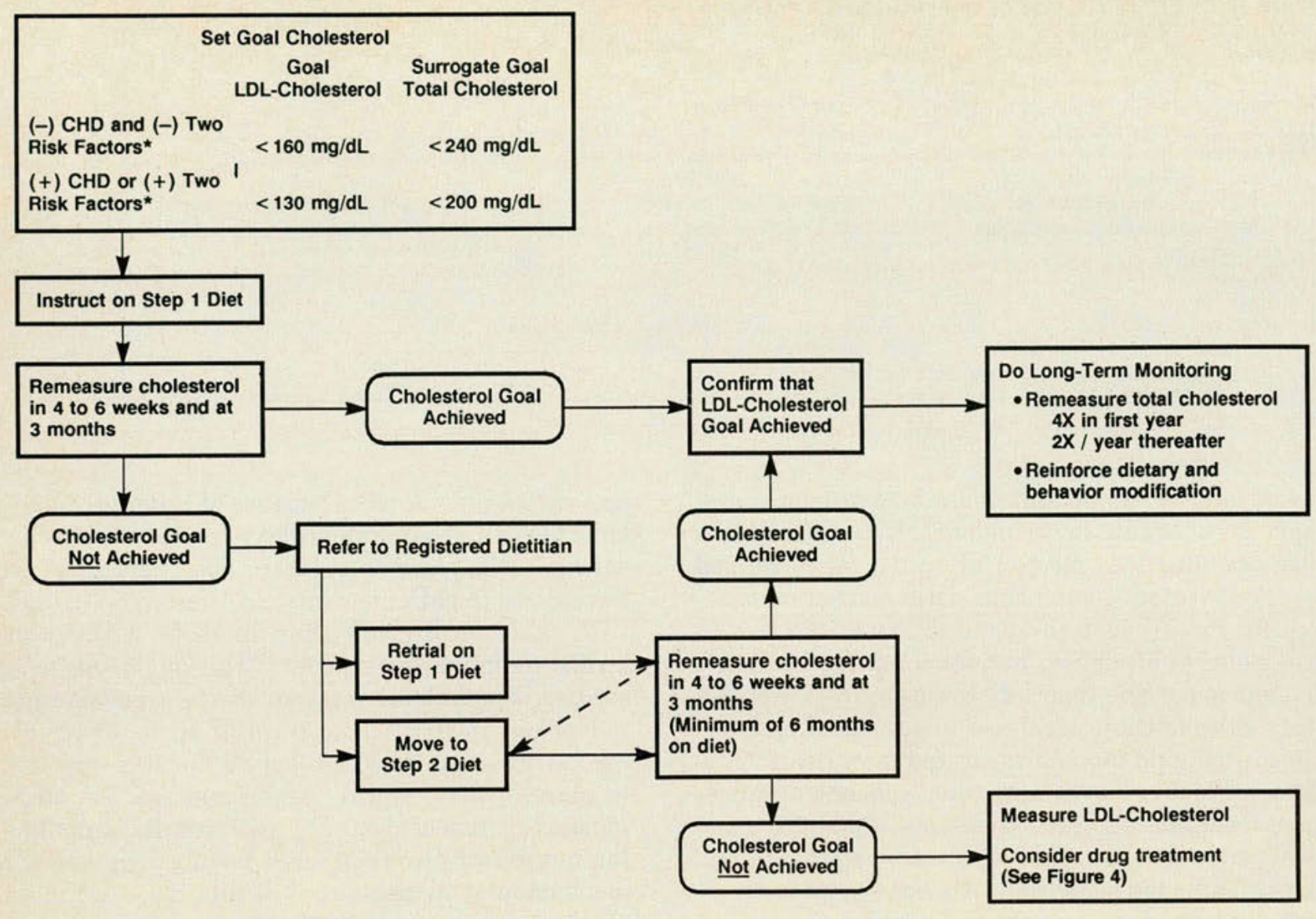

Fig 3. Dietary treatment. Asterisk indicates one of which can be male sex. Reprinted from Archives of Internal Medicine (1988;36-69).

least six months of intensive dietary therapy and counseling, despite which the LDL cholesterol level remains high. There are some exceptions. Patients with LDL cholesterol levels above $225 \mathrm{mg} / \mathrm{dL}$ are unlikely to achieve adequate cholesterol reductions with diet alone. Those with definite CHD may need more immediate and substantial response. Even in these patients, however, three months of dietary therapy is the least that will give an adequate baseline by which to judge drug response. It is also assumed that other nondrug routes to lowering cholesterol values, such as losing weight and increasing exercise, have been fully exploited. Whatever the length of the diet trial, intensive dietary therapy is continued concurrently with the pharmacologic approach selected.

The cutpoints for drug therapy, once there has been a six-month trial of diet alone in which the LDL cholesterol goal was not reached, are $190 \mathrm{mg} /$ $\mathrm{dL}$ in patients without definite $\mathrm{CHD}$ or other CHD risk factors, and $160 \mathrm{mg} / \mathrm{dL}$ in those with definite CHD or at least two other risk factors (Fig 4). The report emphasizes the need for judgment in using these cutpoints. For example, men with cholesterol levels between the two values who have any major risk factor besides male sex usually should be started on a suitable drug regimen, but women with LDL cholesterol levels in this range should be treated more conservatively, with two risk factors required before drug treatment is chosen. Once again, the minimum allowable therapeutic target levels of LDL cholesterol are $<160 \mathrm{mg} / \mathrm{dL}$ in the first group and $<130 \mathrm{mg} / \mathrm{dL}$ in the second, but values as low as $100 \mathrm{mg} / \mathrm{dL}$ may prove to be ideal, especially in patients bearing other risk factors.

The major drugs considered in the guidelines are the bile-acid sequestrants (colestyramine and colestipol hydrochloride) nicotinic acid, HMG CoA reductase inhibitors (lovastatin), gemfibrozil, and probucol. The sequestrants and nicotinic acid are considered the drugs of first choice, primarily because of their long safety records and proved efficacy in lowering LDL cholesterol. Nicotinic acid is recommended when there is concomitant hypertriglyceridemia, because the other two agents sometimes can exacerbate this disorder. Lovastatin is very effective in lowering LDL cholesterol levels and, unlike the first three drugs, it is well tolerated and easy to administer. However, it is not classed as a drug of first choice because it has not been in 


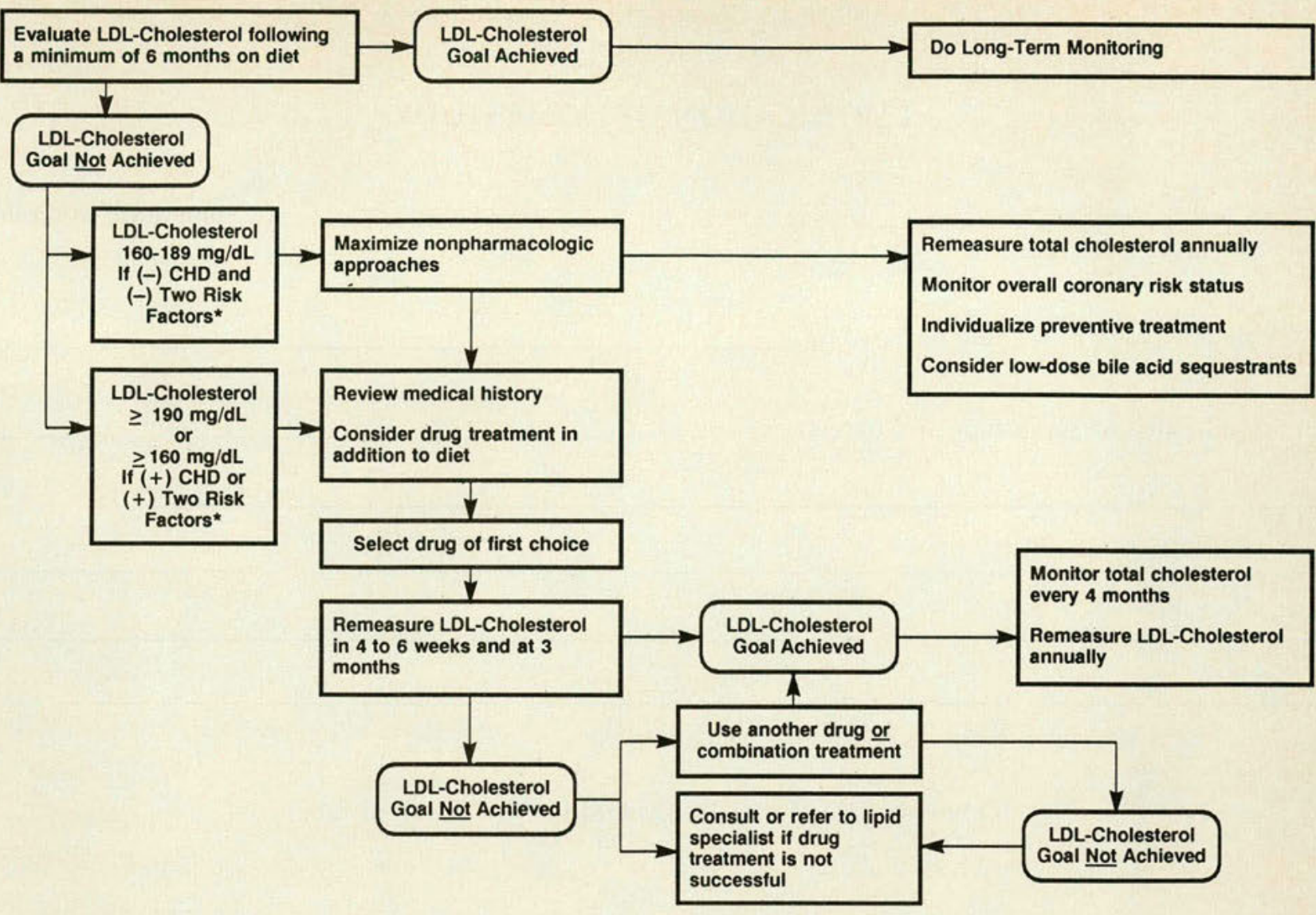

Fig 4. Drug treatment. Asterisk indicates one of which can be male sex. Reprinted from Archives of Internal Medicine (1988;36-69).

use long enough to establish a safety record, nor have its effects on CHD end-points been completely established. The report discusses combination drug treatments for cases in which a single drug plus diet is inadequate.

\section{Comment}

This article has touched only the highlights of a complex and detailed document that has tremendous implications for the practice of medicine in the near future. Any physician likely to be required to treat hypercholesterolemia or the other major CHD risk factors must become thoroughly familiar with these guidelines. He or she may or may not agree with all of their recommendations, but they will be central to the nation's management of this very costly disorder for years to come.

1. Rifkind, BM: Lipid Research Clinics Coronary Primary Prevention Trial: Results and implications. Am J Cardiol 1984;54:30C-34C.

2. The Lipid Research Clinics Program: The Lipid Research Clinics Coronary Primary Prevention Trial results: I. Reduction in incidence of coronary heart disease. JAMA 1984;251:351-364.

3. Lipid Research Clinics Program: The Lipid Research Clinics Coronary Primary Prevention Trial results: II. The relationship of reduction in incidence of coronary heart disease to cholesterol lowering. JAMA $1984 ; 251: 365-374$
4. National Institutes of Health Office of Medical Applications of Research: Lowering blood cholesterol to prevent heart disease. JAMA 1985;253:2080-2086.

5. Report of the National Cholesterol Education Program expert panel on detection, evaluation, and treatment of high blood cholesterol in adults. Arch Int Med 1988;148:36-69.

6. Brown WV: The cholesterol hypothesis and the value of intervention JAOA 1988;88:859-864.

7. Kreisberg RA: Hypercholesterolemia: Dietary and pharmacotherapy Hosp Pract 1987;22:197-201,206-207,211-212.

8. Castelli WP: The triglyceride issue: A view from Framingham. Am Heart $J$ 1986;112:432-437.

9. Consensus Development Panel: Treatment of hypertriglyceridemia. JAMA 1984;251:1196-1200.

10. Kannel WB, Neaton JD, Wentworth D, et al: Overall and coronary heart disease mortality rates in relation to major risk factors in 325,348 men screened for the MRFIT. Am Heart $J$ 1986;112:825-836.

11. Lerner DJ, Kannel WB: Patterns of coronary-heart-disease morbidity and mortality in the sexes: A 26-year follow-up of the Framingham population. Am Heart $J$ 1986;111:383-90.

12. Feinlieb M, Gillum RF: Coronary heart disease in the elderly: The magnitude of the problem in the United States, in Wenger NK, Furberg CD, Pitt E (eds): Coronary Heart Disease in the Elderly. New York, Elsevier Science Publishing Co, 1986, pp 29-59.

13. Hunninghake DB: Current and new therapy for hypercholesterolemia. JAOA 1988;88:865-869.

Dr Voss, an internist, is the AOA representative on the $\mathrm{Na}$ tional Coordinating Committee of the National Cholesterol Educational Program.

Dr Voss, 1111 Madison St, Jefferson City, MO 65101. 